NOTICE: This is the author's version of a work that was accepted for publication in Appetite. Changes resulting from the publishing process, such as peer review, editing, corrections, structural formatting, and other quality control mechanisms may not be reflected in this document. Changes may have been made to this work since it was submitted for publication. A definitive version was subsequently published in Appetite [57, 1, 2011] DOI 10.1016/j.appet.2011.03.015 


\section{Accepted Manuscript}

Title: Omega-3 Fatty Acids: What Consumers Need to Know

Authors: Alexandra McManus, Margaret Merga, Wendy

Newton

PII:

S0195-6663(11)00119-X

DOI: doi:10.1016/j.appet.2011.03.015

Reference:

APPET 1201

To appear in:

Received date: $\quad 1-10-2010$

Revised date: $\quad 3-2-2011$

Accepted date: $\quad$ 28-3-2011

Please cite this article as: McManus, A., Merga, M., \& Newton, W., Omega-3 Fatty Acids: What Consumers Need to Know, Appetite (2010), doi:10.1016/j.appet.2011.03.015

This is a PDF file of an unedited manuscript that has been accepted for publication. As a service to our customers we are providing this early version of the manuscript. The manuscript will undergo copyediting, typesetting, and review of the resulting proof before it is published in its final form. Please note that during the production process errors may be discovered which could affect the content, and all legal disclaimers that apply to the journal pertain. 
Short Communication

\title{
Omega-3 Fatty Acids: What Consumers Need to Know
}

\author{
Alexandra McManus ${ }^{\mathrm{a}}$, Margaret Merga ${ }^{\mathrm{a}}$ and Wendy Newton ${ }^{\mathrm{a}}$ \\ ${ }^{a}$ Centre of Excellence for Science, Seafood \& Health, Curtin University, \\ GPO Box U1987, Perth, WA, 6845 Australia
}

Authors:

Associate Professor Alexandra McManus (corresponding author)

Director

Centre of Excellence for Science, Seafood \& Health, Curtin University

7 Parker Place, Technology Park, WA, 6102 Australia

GPO Box U1987, Perth, WA, 6845 Australia

Telephone: $\quad+61892662115$

Facsimile: $\quad+61892662508$

Email: $\quad$ A.McManus@curtin.edu.au

Margaret Merga

Senior Education Specialist

Centre of Excellence for Science, Seafood \& Health, Curtin University

7 Parker Place, Technology Park, WA, 6102 Australia

GPO Box U1987, Perth, WA, 6845 Australia

Telephone: $\quad+61892669760$

Facsimile: $\quad+61892662508$

Email: $\quad$ M.Merga@curtin.edu.au

Doctor Wendy Newton

Post Doctoral Scientist

Centre of Excellence for Science, Seafood \& Health, Curtin University

7 Parker Place, Technology Park, WA, 6102 Australia

GPO Box U1987, Perth, WA, 6845 Australia

Telephone: $\quad+61892662042$

Facsimile: +61892662508

Email: $\quad$ W.Newton@curtin.edu.au 


\begin{abstract}
The general public is increasingly aware of the health benefits associated with consumption of omega-3 fatty acids. While evidence of health benefits continues to mount, the underlying science is complex. Omega-3 fatty acids vary in their physiological efficacy.
\end{abstract}

Consumers are typically unaware of differences in the efficacy of different omega-3 fatty acids and this lack of knowledge can result in consumers being mislead within the marketplace. There is a need for consumers to be educated about the distinctions between omega-3 fatty acids. In the interim consumers remain at risk of purchasing premium fortified products and supplements that will not correspond to their desired health outcomes. This paper summarises the current understanding of fatty acid physiological metabolism and interaction for the purpose of highlighting this complex and multifaceted concern.

Keywords: omega-3, seafood, fish, consumer, fortification, enrichment, supplementation

\title{
Introduction
}

Omega-3 long chain polyunsaturated fatty acids (n-3 LC-PUFAs) are currently enjoying considerable commercial popularity as a result of consistent findings of health benefits associated with their consumption. The Australian marketplace has seen a proliferation of food products fortified with n-3 LC-PUFAs, as well as phenomenal growth in pharmaceutical omega-3 supplements. 
Globally, the market for n-3 LC-PUFAs supplements is estimated at over US\$700 million with growth rates of $8 \%$ per annum (Clough, 2008). Australia and Spain are regarded as international lead markets for omega-3 products and supplements with Australian consumers purported to have one of the highest levels of consumer awareness of omega-3 fatty acids in the world (Mellentin, 2008). Strong growth continues in the n-3 LC-PUFA supplement market while n-3 LC-PUFAs fortified food products have followed the trend previously demonstrated by food products fortified with plant sterols. Consumer hype and new product proliferation within the mass market has settled to provide a niche market; infant formula is an exception where n-3 LC-PUFAs are virtually a standard category ingredient (Mellentin, 2008).

Positive health outcomes associated with consumption of n-3 LC-PUFAs have been demonstrated in the areas of infant development, cardiovascular disease, platelet aggregation, hypertension, hyperlipidemia, cancer, dementia and Alzheimer's disease, depression, and inflammation (McManus, Howieson, \& Nicholson, 2009; Riediger, Othman, Suh, \& Moghadasian, 2009; Ruxton \& Derbyshire, 2009). Increasing tissue levels of n-3 LC-PUFA at a population level may reduce the incidence of those chronic diseases responsible for the largest portion of the global burden of disease (Hibbeln, Nieminen, Blasbalg, Riggs, \& Lands, 2006).

Research into the health benefits associated with n-3 LC-PUFAs does not follow a standard protocol. Variations in aspects of research design such sample size, subject characteristics, duration of study, n-3 LC-PUFA dosages and form, plasma measurement and analysis influence research results and conclusions. Researchers have found low level n-3 LC-PUFA supplementation in patients receiving ongoing cardiovascular treatments did not reduce the rate of cardiovascular events (Kromhout, 
Gitay, \& Geleijnse, 2010). Higher supplementation doses may have produced a different conclusion. Researchers reviewing the influence of n-3 LC-PUFAs on mortality, cardiovascular disease and cancer state that adjustment for lifestyle factors could be improved in cohort studies (Hooper et al., 2006). Despite the undisputable presence of research papers having mixed polarity, there is a vast and mounting quantity of robust research supporting the positive health impact of n-3 LC-PUFAs.

While there are three n-3 LC-PUFAs commonly found in food, evidence of health benefits is predominantly associated with the marine sourced n-3 LC-PUFAs: eicosapentaenoic acid (EPA) and docosahexaenoic acid (DHA). Alpha-linoleic acid (ALA) is a non marine sourced n-3 LC-PUFA and occurs in seed crops such as canola. The strongest associations between n-3 LC-PUFAs and health have been demonstrated with DHA intake and more recently with a dietary pattern that includes seafood. This indicates that marine based n-3 LC-PUFAs have a greater positive impact on health than non marine sourced n-3 LC-PUFAs. For instance, a review of the efficacy of n-3 LCPUFAs in primary and secondary prevention studies on cardiovascular disease revealed that consumption of n-3 LC-PUFAs from fish or fish oil consumption, but not consumption of ALA, improved rates of all-cause mortality, cardiac and sudden death (Wang et al., 2006).

Consumers are not aware of the relative efficacy of the alternative n-3 LC-PUFA forms from both dietary and supplemental sources. Efficacy of n-3 LC-PUFAs is influenced by n-3 LC-PUFA type; additionally in supplements or fortified foods, efficacy will be influenced by the food matrix, processing and storage. This paper provides an overview of the complexity and interaction of n-3 LC-PUFAs in order to highlight the 
enormous potential for consumer misunderstanding in the marketing of omega-3 fatty acid fortified food and supplements.

\section{Omega 3 Fatty Acids}

Naturally occurring lipids are made up of aliphatic monocarboxylic acids (fatty acids). Fatty acids are named in the hydrocarbon manner reflecting the length of the carbon chain and the number and location of double bonds within the molecule (Nawar, 1985). The terminal carboxyl group is designated as carbon number one in the backbone of the molecule. Conversely however, when reference is made to an omega numbered fatty acid, the omega number refers to the carbon molecule adjacent to a double bond numbered from the methyl end of the molecule. DHA (22:6n-3) for example, is a molecule with a backbone 22 carbon atoms in length and contains 6 double bonds with the first double bond occurring on carbon 3 from the methyl end. Figure 1 displays the three omega-3 fatty acids commonly found in food.

\section{Physiological Interactions}

Despite the structural similarity of n-3 LC-PUFAs, human metabolism and physiological function of these fatty acids are divergent. Humans do not possess the enzymes necessary to synthesise ALA and as such ALA is an essential fatty acid in human diets (Goyens, Spilker, Zock, Katan, \& Mensink, 2006). The human body can however convert ALA into DHA or EPA. Rates of conversion of ALA to EPA have been observed to be as high as $8 \%$ while conversion to DHA is very limited (Goyens et al., 2006). The impact of conversion rates becomes clear when comparing the equivalency of different n-3 LC-PUFAs. Ingested preformed EPA is equivalent to ingested ALA at a ratio of 15:1 (Brenna, Salem Jr, Sinclair, \& Cunnane, 2009). 
Investigations involving plasma lipid profiles can be challenging as measurements at any one time represent a state of physiological flux at the time of sample collection. Research investigating the balance of fatty acids in plasma therefore must be cognisant of the dynamic nature of fatty acid balance. Plasma lipid profiles at any one point in time are the product of ingested n-3 LC-PUFAs, conversion rates and the PUFA profile of the background diet.

As n-3 LC-PUFAs contain a carbon-carbon double bond between the third and fourth carbon from the methyl end of the fatty acid molecule, $n-6$ and n-9 LC-PUFAs contain a double bond on the sixth and ninth carbon respectively. An antagonistic relationship exists between n-3 and n-6 LC-PUFAs with regard to biological status (Brenna et al., 2009). Ingested n-3 LC-PUFAs compete with n-6 LC-PUFAs for enzymes essential for conversion of ALA to EPA or DHA (Simopoulos, 2008). Major dietary n-6 LC-PUFAs are linoleic acid (LA , 18:2n-6) and arachodinic acid (AA, 20:4n-6). These PUFAs occur naturally in seed oils and are a significant component of the western type dietary pattern. The western diet is estimated to have a ratio of n-6 to n-3 LC-PUFA of between 15:1 and 16.7:1 (Simopoulos, 2008). High dietary levels of LA have been found to result in increased tissue AA and reduced tissue n-3 LC-PUFAs as well as nervous system alterations (Brenna et al., 2009). Reducing the ratio of n-6 to n-3 LCPUFA to $4: 1$ has been found to decrease to reduce total mortality by $70 \%$ in secondary prevention of cardiovascular disease; a ratio of 5:1 is beneficial for asthma; $2-3: 1$ reduces rheumatoid arthritis inflammation and a ratio of $2.5: 1$ reduces colorectal cancer cell proliferation (Simopoulos, 2008). It is recommended that the ratio of n-6 to n-3 LCPUFA not exceed 4:1 (Garg, Wood, Singh, \& Moughan, 2006). 
Dietary fatty acid profile and interactions define effective EPA and DHA conversion. Researchers estimate that large reductions in dietary n-6 LC-PUFA intake could reduce dietary n-3 LC-PUFA requirements by as much as 90\% (Hibbeln et al., 2006). Optimal EPA synthesis is noted in diets low in LA and increases in DHA synthesis are observed in diets high in ALA (Goyens et al., 2006). DHA synthesis is 'extremely limited' and a low LA, high ALA diet will not significantly increase plasma DHA as would a moderate intake of seafood or fish oils. Consumption of seafood remains the only demonstrable pathway to significantly increased DHA status. Exchange of LA for ALA to enhance fatty acid plasma profiles is recommended as a less effective option for individuals that do not consume seafood (Goyens et al., 2006).

Individuals differ in their ability to metabolise nutrients and in their genetic predisposition to chronic disease. The fields of nutrigenomics and nutrigenetics encompass the influence of diet on the genome and the influence of phenotypical genetic differences on individual responses to diet (Mutch, Wahli, \& Williamson, 2005). Genetic information is eminent within the study of chronic disease; increased risk of chronic disease can identify individuals that will benefit from preventative dietary intervention (Omenn, 2009).

Over the last 150 years, the human westernised diet has undergone unprecedented rapid change. In evolutionary terms the typical human diet contained an n-6 to n-3 LC-PUFA ratio of approximately one (Simopoulos, 2006). Despite individual genetic differences, analysis of international data including apparent dietary intakes and relative risks of mortality and morbidity for 38 countries found that the total mortality disease burden potentially attributable to deficiency in n-3 LC-PUFAs was $20.8 \%$ for males and $31.5 \%$ for females (Hibbeln et al., 2006); specific conditions attributable to n-3 LC-PUFA 
deficiency were as high as $41.2 \%$ (males) and $42.5 \%$ (females) for coronary heart disease mortality, $28.4 \%$ for homicide mortality and $98.5 \%$ for major depression. The researchers concluded that reduction of n-6 LC-PUFA consumption could reduce the dietary requirement for n-3 LC-PUFA to one tenth of its current level.

\section{Fortification in Food}

Codex Alimentarius defines food fortification or enrichment as the addition of an essential nutrient(s) to food that may or may not naturally contain the nutrient in order to address a nutrient deficiency within a populace. Enrichment of foods with n-3 LCPUFAs is regarded as a way of increasing dietary intake of these fatty acids in order to reduce levels of risk of 'life-style' diseases and cardiovascular disease in particular (Kolanowski, 2005). It is necessary for levels of fortification to provide significant contributions toward recommended dietary n-3 LC-PUFA intakes within the portion size that will be consumed by the individual.

Efficacy of n-3 LC-PUFAs in fortified foods will depend upon fortification with those n-3 LC-PUFA forms that provide the highest level of health benefit. The health benefits associated with marine sourced n-3 LC PUFAs have been widely publicised and are likely the motivation for consumer purchase of fortified foods. Fortification with fish oil, EPA or DHA will provide the health benefits consumers expect and greater physiological benefit than fortification with ALA. The quality of fish oil used, type of food fortified and the nutrient profile of the food will also influence the physiological function of n-3 LC-PUFAs (Kolanowski, 2005).

A major challenge in the development of enriched food products is presented by the multiple acceptance criteria to be achieved despite the addition of an active ingredient: 
product freshness, sensory characteristics, appearance, storage conditions, ease of preparation and safety standards (Drusch \& Mannino, 2009). Fish oil is highly susceptible to oxidation. Oxidation of n-3 LC-PUFAs can produce toxic peroxides and other by-products and as such it is recommended that well refined and stabilised fish oil is sourced for the purpose of food enrichment (Kolanowski, 2005). Formulation, processing, packaging, shelf life and storage conditions of foods fortified with n-3 LCPUFAs are critical for both bioavailability and oxidative stability. The influence of these factors is complex and multifaceted. Despite the known influence of temperature on oxidation, greater oxidative stability of fish oil fortified energy bars has been demonstrated through addition of oil during mixing of the dough rather than coating bars after baking (Nielsen and Jacobsen, 2009).

The form in which the fish oil is added, such as neat, within an emulsion or microencapsulated will further influence bioavailability and oxidative stability. Emulsification of fish oil prior addition to product matrix and use of modified atmosphere packaging increase the oxidate stability of fish oils (Nielsen and Jacobsen, 2009). Neither technique was as effective at improving oxidative stability as the use of a microencapsulated form of fish oil.

Foods fortified with n-3 LC-PUFAs are thought to have similar bioavailability of fatty acids as capsule supplements (Kolanowski, 2005). Bioequivalence has been demonstrated for algal oil DHA delivered in capsules and fortified snack bars (Arterburn et al., 2007). Absorption of EPA and DHA from fortified foods has however been observed to occur at a faster rate than absorption from supplement capsules (Schram et al., 2007). Further, the nature of the food matrix will influence the rate of 
absorption; absorption of EPA and DHA from a yoghurt drink has been found to be faster than from a fitness bar.

\section{Supplementation}

Recent research has revealed clear differences in the bioavailability of alternate forms of concentrated fish oil (Dyerberg, Madsen, Moller, Aardestrup, \& Schmidt, 2010). Reesterified triglyceride forms were identified as having superior bioavailability; free fatty acids have moderate bioavailability (comparable to natural fish oils or triglycerides) and ethyl esters to have lower bioavailability (Dyerberg et al., 2010). Further investigations have been conducted into the bioavailability of oils within gelatine capsules, microencapsulated by spray drying milk protein and gelatine-based compounds and multiple shell microencapsulation (Drusch \& Mannino, 2009). Microencapsulated fish oil supplements are considered superior though researchers note that supplements should be consumed within a diet high in monounsaturated fatty acids and low in n-6 LC-PUFAs (Garg et al., 2006). Additionally, supplements should be consumed with a meal as absorption of n-3 LC-PUFAs within a food matrix is enhanced. The presence of the food matrix augments the rate of entry into intestinal mucosal cells (Garg et al., 2006).

\section{Discussion}

Debate about the role of fortified food and nutrient supplements for human health continues amidst emerging scientific knowledge and marketplace reactivity. The advocates of n-3 LC-PUFA enriched foods and supplements accurately note that given low metabolic conversion of other dietary n-3 LC-PUFA to DHA, individuals that do not consume seafood, muscle or organ meats will not achieve recommended DHA 
intakes or status without fortification or supplementation (Garg et al., 2006). In addition, individuals that are at risk of certain chronic diseases such as cardiovascular disease have very high recommended daily doses of $1 \mathrm{~g}$ of n-3 LC-PUFAs (Garg et al., 2006). Without dietary inclusion of n-3 LC-PUFA fortified foods or supplements, these individuals would be unlikely to achieve this recommended level of intake.

Increasingly however, research into positive health outcomes and nutrition is revealing the strongest evidence with relation to dietary patterns rather than to individual nutrients (Lichtenstein \& Russell, 2005). The use of a whole diet approach is further supported by the complexity of nutrient requirements and interactions and the disappointing results of many single nutrient interventions (Lichtenstein \& Russell, 2005). The nutritional benefit of a diet rich in seafood and oily fish in particular extends beyond n-3 LC-PUFAs content. Fish, as a whole food, is an excellent source of lean protein and other vitamins and minerals including vitamins A and D, B group vitamins, iron, zinc, selenium and iodine (Hostenkamp \& Sorensen, 2009).

Liberal food fortification strategies can change perceptions around what constitutes healthy food (Meltzer, Aro, Andersen, Koch, \& Alexander, 2003). Risk analysis of food product fortification raises the issue of overweight, obesity and chronic disease in the developing world and highlights the need for discussion and regulation arising from the possibility that fortification may actually promote consumption of an unhealthy or unbalanced diet (Meltzer et al., 2003). Likewise, consumer confusion about the messages conveyed in the promotion of supplements can have a potentially negative impact on diet and lifestyle factors and support the need for a food-based diet approach to achieve optimal public health outcomes (Lichtenstein \& Russell, 2005). There is an obvious exception in the need for targeted supplementation to specific population 
segments eg. Vitamin D and calcium supplements for elderly patients (Lichtenstein \& Russell, 2005).

A further cause for concern is self administration of supplements without awareness of nutrient or drug interactions. The cardiovascular benefits associated with EPA and DHA are associated with reduced coagulatability of blood; self administration of $n-3$ LC-PUFAs by a person taking anticoagulants could result in a bleeding tendency (Mason, 2009). Additionally, the oxidative stability of n-3 LC-PUFAs in supplements and fortified foods requires ongoing research.

There is a clear need for the public to be educated about the relative efficacy of n-3 LCPUFAs. The commercial popularity of n-3 LC-PUFAs has its roots in positive research linking EPA and DHA to health benefits. The greatest health benefit can be obtained from consumption of adequate dietary n-3 LC-PUFAs within a food matrix. The most direct, bioavailable, uncomplicated and unadulterated sources of efficacious preformed EPA and DHA are fish and seafood. A $100 \mathrm{~g}$ serving of sardines provides $0.98-1.7 \mathrm{~g}$ of combined DHA and EPA, herring 1.71-1.78g, and lobster 0.07-0.41g (Kris-Etherton, Harris \& Appel, 2002).

Consumers require access to information about the benefits of consuming fish and seafood, the relative efficacy of different n-3 LC-PUFAs and the interaction of other dietary fatty acids. Almost half of Australians do not have adequate health literacy to understand health information and instructions (Australian Bureau of Statistics, 2006). Similar levels have been identified internationally. In the US, $28.7 \%$ of parents have been identified as having basic or below basic health literacy (Yin et al., 2009); 30\% of Taiwanese adults have marginal or inadequate health literacy despite over $20 \%$ of the 
population possessing tertiary qualifications (Lee, Tsai \& Kuo, 2010). The challenge for public health professionals transpires in communication of complicated and evolving scientific knowledge with simple and straightforward messages that have widespread availability.

\section{Conclusion}

It is thought that consumers are largely unaware of differences in efficacy of n-3 LCPUFAs and may assume that the much publicised health benefits of DHA and EPA may apply to other omega 3 and / or omega 6 fatty acids. Physiological efficacy of alternate n-3 LC-PUFAs is different.

Demonstrable evidence of health benefits associated with consumption of marine sourced n-3 LC-PUFAs, DHA and EPA, is strong and continues to mount. There is a need for a straightforward consumer communication strategy to inform consumers that consumption of diet that includes moderate levels of seafood is the simplest and best way to obtain the health benefits associated with n-3 LC-PUFA consumption.

Health professionals can reduce the propensity for consumer misinformation by communicating the superior efficacy of marine sourced n-3 LC-PUFAs and the beneficial role of seafood within a balanced diet. Foods enriched with n-3 LC-PUFAs and supplements can help those individuals that have recommended daily intakes of $n-3$ LC-PUFAs that are difficult to meet with diet alone or individuals that do not consume seafood. 


\section{REFERENCES}

Arterburn, L.M., Oken, H.A., Hoffamn, J.P., Bailey-Hall, E., Chung, G., Rom, D., et al. (2007). Bioequivalence of Docosahexaenoic Acid of Different Algal Oils in Capsules and in a DHA-Fortified Food. Lipids, 42, 1011-1024.

Australian Bureau of Statistics. (2006). Health Literacy, Australia. Retrieved 27th April 2010 ,

Brenna, J. T., Salem Jr, N., Sinclair, A. J., \& Cunnane, S. C. (2009). $\alpha$-Linoleic Acid Supplementation and Conversion to n-3 Long-Chain Polyunsaturated Fatty Acids in Humans. Prostaglandins, Leukotrienes and Essential Fatty Acids, 80, 85-91.

Clough, P. (2008). Marketing Omega-3 Products as Nutraceuticals. International Symposium on Fatty Acids - Opportunities for Health Education and Investment, Tunis, 19(3), 233-234.

Drusch, S., \& Mannino, S. (2009). Patent-based Review on Industrial Approaches for the Microencapsulation of Oils Rich in Polyunsaturated Fatty Acids. Trends in Food Science and Technology, 20, 237-244.

Dyerberg, J., Madsen, P., Moller, J. M., Aardestrup, I., \& E.B., S. (2010). Bioavailability of Marine n-3 Fatty Acid Formulations. Prostaglandins, Leukotrienes and Essential Fatty Acids, In Press. 
Garg, M. L., Wood, L. G., Singh, H., \& Moughan, P. J. (2006). Means of Delivering Recommended Levels of Long Chain n-3 Polyunsaturated Fatty Acids in Human Diets. Journal of Food Science, 71(5), R66-R71.

Goyens, P. L. L., Spilker, M. E., Zock, P. L., Katan, M. B., \& Mensink, R. P. (2006). Conversion of a-Linoleic Acid in Humans is Influenced by the Absolute Amounts of aLinoleic Acid and Linoleic Acid in the Diet and not by Their Ratio. American Journal of Clinical Nutrition, 84, 44-53.

Hibbeln, J.R., Nieminen, L.R.G., Blasbalg, T.L., Riggs, J.A., \& Lands, W.E.M. (2006). Healthy intakes of n-3 and n-6 fatty acids: estimations considering worldwide diversity. American Journal of Clinical Nutrition, 83(suppl), 1483S-1493S.

Hostenkamp, G., \& Sorensen, J. (2009). Are fish eaters healthier and do they consumer less health-care resources? Public Health Nutrition, 13(4), 453-460.

Hooper, L., Thompson, R.L., Harrison, R.A., Summerbell, C.D., Ness, A.R., Moore, H.J., et al. (2006). Risks and benefits of omega 3 fats for mortality, cardiovascular disease, and cancer: systematic review. British Medical Journal Online, doi:10.1136/bmj.38755.366331.2F. 
Kolanowski, W. (2005). Bioavailability of Omega-3 PUFA from Foods Enriched with Fish Oil - A Mini Review. Polish Journal of Food and Nutrition Sciences, 14/55(4), $335-340$.

Kris-Etherton, P.M., Harris, W.S., \& Appel, L.J. (2002). Fish Consumption, Fish Oil, Omega-3 Fatty Acids, and Cardiovascular Disease. Circulation, 106, 2747-2757.

Kromhout, D., Giltay, E.J., \& Geleijnse, J.M. (2010). n-3 Fatty Acids and cardiovascular Events after Myocardial Infarction. The New England Journal of Medicine, 363(21), 2015-2026.

Lee, S.-H.D., Tsai, T.-I., \& Kuo, K.N. (2010). Health literacy, health status, and healthcare utilization of taiwanese adults: results from a national survey. Biomed Central Public Health, 10, 614-622.

Lichtenstein, A. H., \& Russell, R. M. (2005). Essential Nutrients: Food or Supplements? Journal of the American Medical Association, 294(3), 351-358.

Mason, P. (2009). Important Drug-nutrient Interactions. Paper presented at the Conference on 'Malnutrition Matters', Cardiff.

McManus, A., Howieson, J., \& Nicholson, C. (2009). Review of literature and resources relating to the health benefit of regular consumption of seafood as part of a 
healthy diet [090101]. Perth: Centre of Excellence for Science, Seafood and Health, Curtin Health Innovation Research Institute, Curtin University of Technology.

Mellentin, J. (2008). End of the line for omega-3? Dairy Industrial Research, 73(9), 1617.

Meltzer, H. M., Aro, A., Andersen, N. L., Koch, B., \& Alexander, J. (2003). Risk Analysis Applied to Food Fortification. Public Health Nutrition, 6(3), 281-290.

Mutch, D.M., Wahli, W., \& Williamson, G. (2005). Nutrigenomics and nutrigenetics:the emerging faces of nutrition. The FASEB Journal, 19, 1602-1616.

Nawar, W. W. (1985). Lipids. In O. R. Fennema (Ed.), Food Chemistry. Second Edition. (pp. 139-244). New York: Marcel Dekker Inc.

Nielsen, N.S., \& Jacobsen, C. (2009). Methods for reducing lipid oxidation in fish-oilenriched energy bars. International Journal of Food Science and Technology, 44, 15361546.

Omenn, G.S. (2010). Overview of the Symposium on Public Health Significance of Genomics and Eco-Genetics. Annual Reviews in Public Health, 31, 1-8. 
Riediger, N. D., Othman, R. A., Suh, M., \& Moghadasian, M. H. (2009). A Sytematic Review of the Roles of n-3 Fatty Acids in Health and Disease Journal of the American Dietetic Association, 109(4), 668-679.

Ruxton, C.H.S. \& Derbyshire, E. (2009). Latest evidence on omega-3 fatty acids and health. Nutrition and Food Science, 39(4), 423-438.

Schram, L.B., Nielsen, C.J., Porsgaard, T., Nielsen, N.S., Holm, R., \& Mu, H. (2007). Food matrices that affect the bioavailability of $(n-3)$ polyunsaturated fatty acids in a single meal study in humans. Food Research International, 40, 1062-1068.

Simopoulos, A.P. (2008). The Importance of the Omega-6/Omega-3 Fatty Acid Ratio in Cardiovascular Disease and other Chronic Diseases. Society for Experimental Biology and Medicine, doi: 10.3181/0711-MR-311 1535-3702/08/2336-0674\$15.00

Simopoulos, A.P. (2006). Evolutionary aspects of diet, the omega-6/omega-3 ratio and genetic variation: nutritional implications for chronic diseases. Biomedicine and Pharmacotherapy, 60, 502-507.

Wang, C., Harris, W. S., Chung, M., Lichtenstein, A. H., Balk, E. M., Kupelnick, B., et al. (2006). n-3 Fatty Acids from Fish or Fish-oil Supplements, but not $\alpha$-Linoleic Acid, Benefit Cardiovascular Disease Outcomes in Primary- and Secondary- Prevention Studies: A Systematic Review. American Journal of Clinical Nutrition, 84, 5-17. 
Yin, H.S., Johnson, M., Mendelsohn, A.L., Abrams, M.A., Sanders, L.M., \& Dreyer, B.P. (2011). The Health Literacy of Parents in the United States: A Nationally Representative Study. Pediatrics, 124, S289-S298. 
Fig 1. Nomeclature of Omega 3 Fatty Acids.

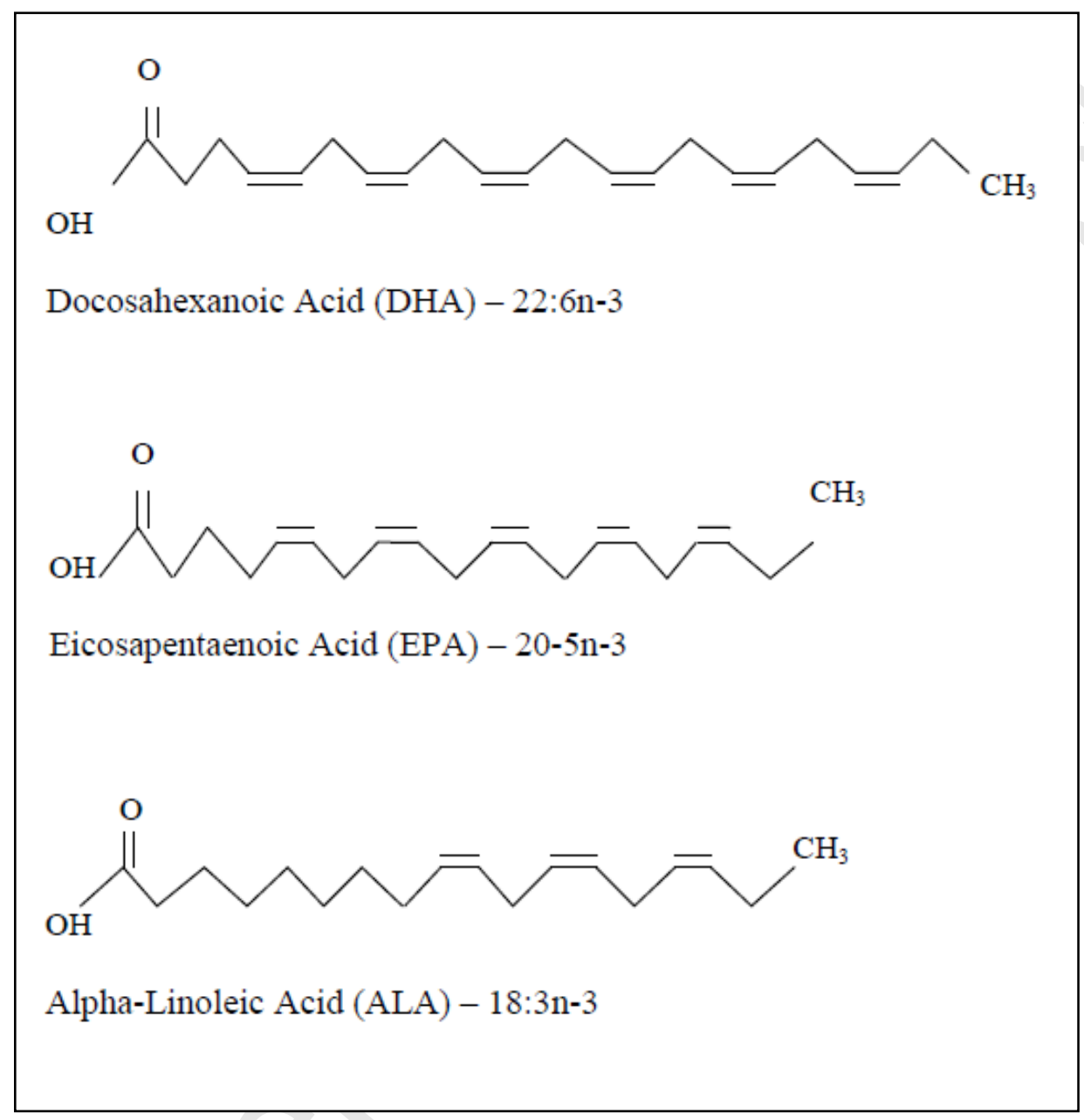

